\title{
Corrigendum to "Ratio of the Dead to Wounded (D/W) Indicators and Associated Factors in Major Earthquakes of America from 1960 to 2011"
}

\author{
A. Ayuso-Alvárez, ${ }^{1}$ K. Alva Chavez, ${ }^{2}$ M. V. Martínez de Aragón, ${ }^{3,4}$ C. R. Ugarte, ${ }^{5}$ \\ J. Donado, ${ }^{1}$ M. S. Rossi, ${ }^{1}$ and Dante R. Culqui ${ }^{1,6}$ \\ ${ }^{1}$ Spanish Field Epidemiology Training Program (SFETP), National Centre for Epidemiology, Carlos III Health Institute, Calle Monforte \\ De Lemos 5, 28029 Madrid, Spain \\ ${ }^{2}$ Institute of Public Goods and Policies (IPP), Spanish Council for Scientific Research (CSIC), Madrid, Spain \\ ${ }^{3}$ Epidemiologic Surveillance Analysis, National Centre for Epidemiology, Carlos III Health Institute, Madrid, Spain \\ ${ }^{4}$ Consortium for Biomedical Research Network in Epidemiology and Public Health (CIBERESP), Madrid, Spain \\ ${ }^{5}$ Pan American Health Organization, World Health Organization, WA, USA \\ ${ }^{6}$ Doctoral Program in Preventive Medicine and Public Health, Autonomous University of Madrid, Madrid, Spain
}

Correspondence should be addressed to M. S. Rossi; rossimarcell@gmail.com

Received 22 March 2015; Accepted 21 April 2015

Copyright (C) 2015 A. Ayuso-Alvárez et al. This is an open access article distributed under the Creative Commons Attribution License, which permits unrestricted use, distribution, and reproduction in any medium, provided the original work is properly cited.

There are some issues in the published paper entitled "Ratio of the Dead to Wounded (D/W) Indicators and Associated Factors in Major Earthquakes of America from 1960 to 2011" which we are very interested to correct through a Corrigendum. We are going to list all the changes that should be included.

(1) In all the places of the paper where the complete name of CRED is needed, it is important to make the correction that CRED is spelled "Centre for Research on the Epidemiology of Disasters."

(2) In Table 5 the text "densidad poblacional" should be replaced by "population density" and the term "IDH" should be replaced by "HDI" which means Human Development Index.

(3) In Section 2.8, because the best definition of number of deaths is that which expresses the number of people dead and missing, we think that is very important to replace the published definition by the previously described one.

(4) In the fourth paragraph in Section 4, where "In this regard, considering the HDI as an operational approach to the quality of housing and an aspect of the vulnerability (the main factor explaining the difference of deaths, wounded, and $\mathrm{D} / \mathrm{W}$ between countries and between regions), the results obtained in our work allow us to suggest that the improvement of HDI was in close relation with the quality of housing. This information is in agreement with some studies that suggest an association between the quality and the height of the buildings and the mortality after an earthquake. These studies have concluded that the higher the quality of housing is, the lower the death toll is $[19,20]$. Furthermore, the greater the height of buildings, the greater the death toll that occurs after an earthquake [21]." is written, in this regard, the differences of HDI could explain the difference of the results obtained when South America and Central America were compared.

(5) At the end of the second paragraph in Section 4, we mention that there is a "concomitant increase in the $\mathrm{D} / \mathrm{W}$ indexes as a consequence of the increase in the HDI." But the results in the table show that, for an increase in the HDI, the $\mathrm{D} / \mathrm{W}$ index would be 
reduced. Because there is a contradiction here, the paragraph should be corrected as follows: "In this regard, a concomitant decrease in the $\mathrm{D} / \mathrm{W}$ indexes was a consequence of the increase in the HDI." Furthermore and according to this, the following part should be deleted: "... which means that the number of people wounded increases with regard to the number of dead people." 

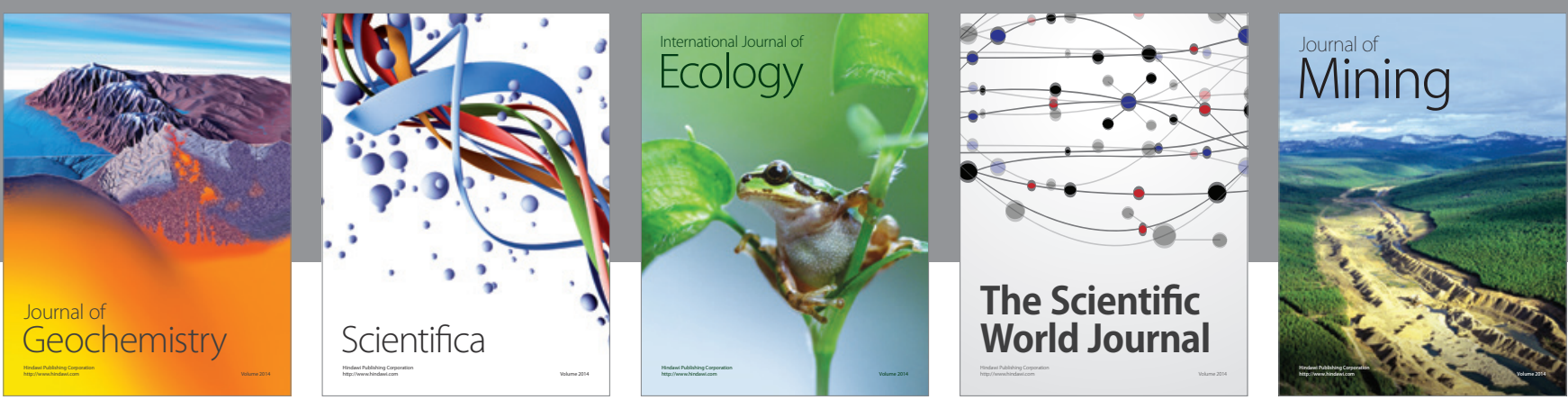

The Scientific World Journal
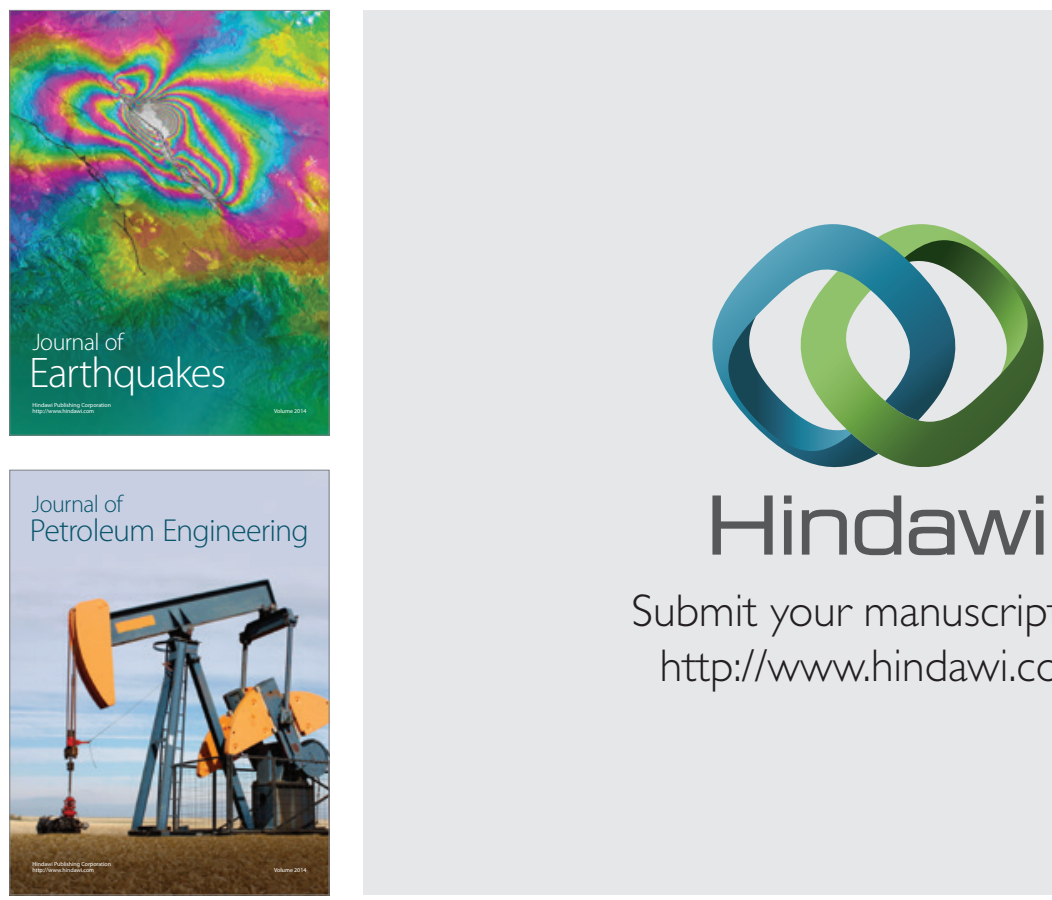

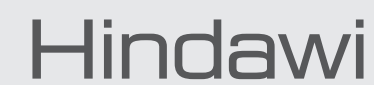

Submit your manuscripts at

http://www.hindawi.com
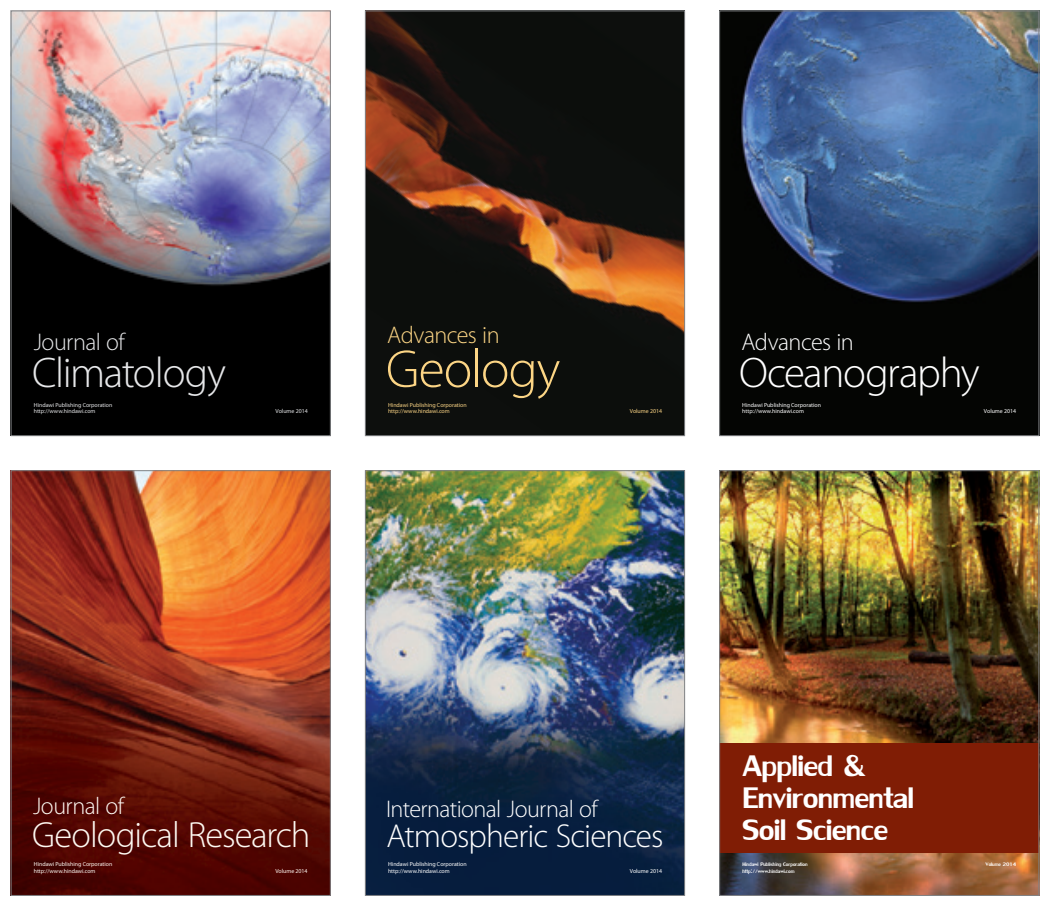
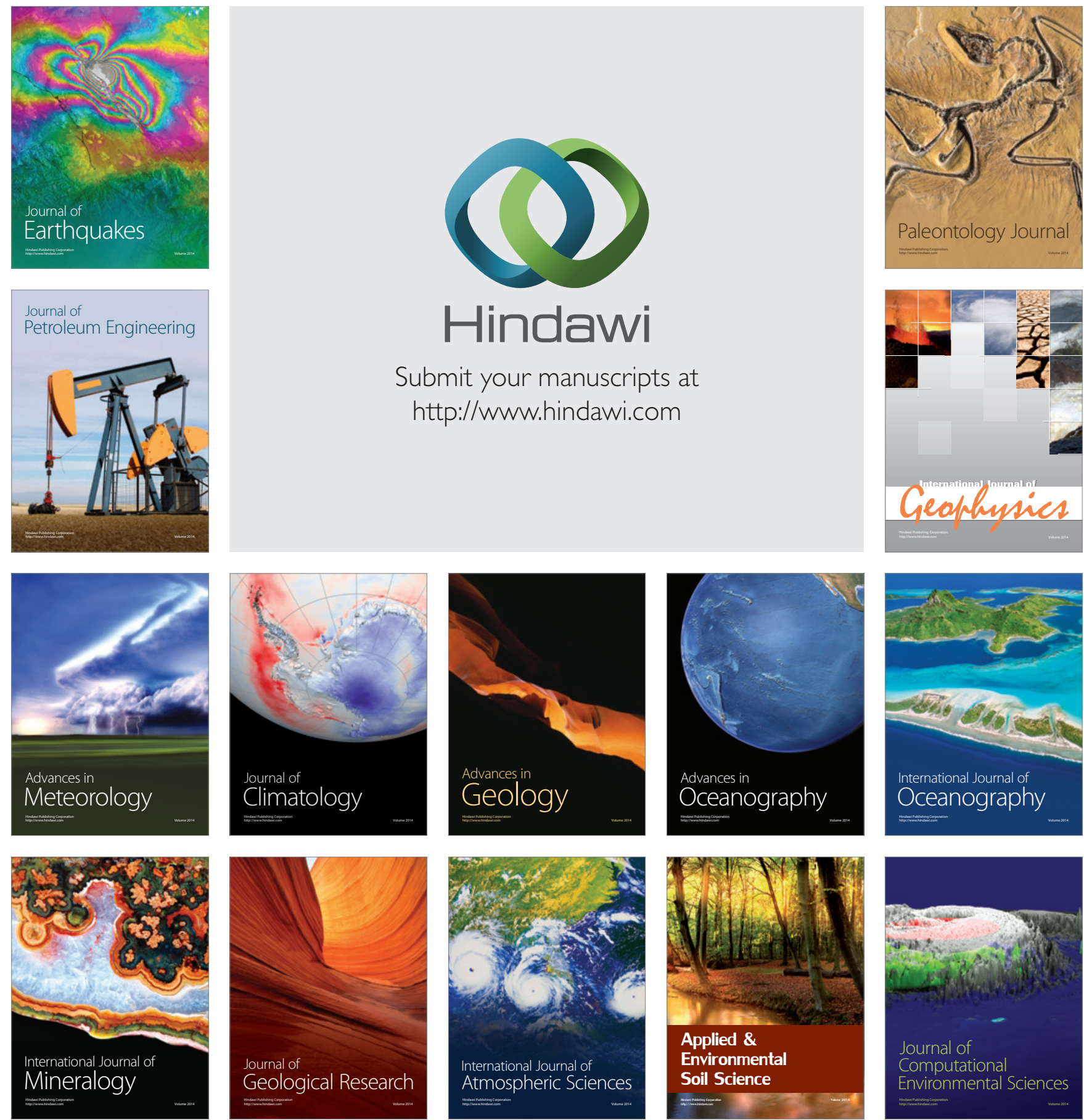